\title{
Effects of TAZ on human dental pulp stem cell proliferation and migration
}

\author{
SONGBO TIAN $^{1,2}$, XIAOCHAO TIAN ${ }^{3}$, YANPING LIU $^{4}$, FUSHENG DONG $^{5}$, \\ JIE WANG $^{2}$, XUQIAN LIU ${ }^{2}$, ZHIYONG ZHANG ${ }^{1}$ and HUIZHEN CHEN ${ }^{1}$
}

\author{
${ }^{1}$ Department of Oral Medicine, Second Hospital of Hebei Medical University; ${ }^{2}$ Department of Oral \\ Pathology, College of Stomatology, Hebei Medical University; ${ }^{3}$ Department of Cardiology and ${ }^{4}$ Physical \\ Examination Center, Second Hospital of Hebei Medical University; ${ }^{5}$ Department of Oral and Maxillofacial \\ Surgery, College of Stomatology, Hebei Medical University, Shijiazhuang, Hebei 050000, P.R. China
}

Received March 12, 2016; Accepted March 1, 2017

DOI: $10.3892 / \mathrm{mmr} .2017 .6550$

\begin{abstract}
Transcriptional coactivator with PDZ-binding motif (TAZ) acts as the key downstream regulatory target in the Hippo signaling pathway. TAZ overexpression has been reported to promote cellular proliferation and induce epithelial-mesenchymal transition in human mammary epithelial cells. However, the effects of TAZ in the regulation of human dental pulp stem cell (hDPSC) proliferation and migration, as well as the molecular mechanisms underlying its actions, remain to be elucidated. The present study demonstrated that TAZ was expressed in hDPSCs. TAZ silencing, following hDPSC transfection with TAZ-specific small interfering (si)RNA (siTAZ), inhibited cellular proliferation and migration in vitro. These effects appeared to be associated with the downregulation of connecting tissue growth factor (CTGF) and cysteine-rich angiogenic inducer (Cyr) 61 expression. Further investigation of the mechanisms underlying the actions of TAZ in hDPSCs revealed that TAZ silencing suppressed CTGF and Cyr61 expression by interfering with transforming growth factor (TGF)- $\beta$ signaling pathways. The present results suggested that TAZ may be implicated in the proliferation and migration of hDPSCs, through the modulation of CTGF and Cyr61 expression via a TGF- $\beta$-dependent signaling pathway.
\end{abstract}

\section{Introduction}

Human dental pulp stem cells (hDPSCs) are derived from human teeth and have garnered attention in stem cell research due to their biological properties, as they are highly clonogenic

Correspondence to: Dr Jie Wang, Department of Oral Pathology, College of Stomatology, Hebei Medical University, 419 Zhongshan Road, Shijiazhuang, Hebei 050000, P.R. China

E-mail: wangjiekouqiang@126.com

Key words: cell proliferation, cell migration, human dental pulp stem cell, transcriptional coactivator with PDZ-binding motif, transforming growth factor- $\beta$ pathway and proliferative. In addition, they are characterized by self-renewal and multi-lineage differentiation $(1,2)$. Therefore, it may be hypothesized that hDPSCs have potential to be used in regenerative medicine, particularly for the development of biologic substitutes, including artificial tissues. High rates of proliferation and migration are critical for cells to be successfully used in regenerative medicine applications (3).

Previous studies have demonstrated that stem cell growth, adhesion and migration may be regulated by several molecules, including transcriptional coactivator with PDZ-binding motif (TAZ) (4-8). TAZ belongs to the family of 14-3-3 cytoplasmic proteins (9). Previous studies have demonstrated its involvement in embryogenesis (10) and the development of several organs, including bone, lung and heart (11-13). TAZ is one of the key downstream effectors of the Hippo signaling pathway, which has been reported to regulate stem cell proliferation, differentiation and survival (14). It has previously been revealed that TAZ promoted the differentiation of mesenchymal stem cells (MSCs) into osteoblastic lineages $(15,16)$, whereas cell-permeable low-molecular-weight protamine-TAZ fusion proteins have been demonstrated to increase the odontogenic and osteogenic differentiation of hDPSCs (17). In addition, TAZ overexpression has been reported to promote cellular proliferation and induce epithelial-mesenchymal transition (10). However, the effects of TAZ on the regulation of hDPSC proliferation and migration, as well as the molecular mechanisms underlying its actions, remain to be elucidated.

In the present study, the effects of TAZ on the proliferation and migration of hDPSCs were investigated. The present results demonstrated that hDPSC proliferation and migration were inhibited following the silencing of TAZ expression. The molecular mechanisms underlying the effects of TAZ knockdown appeared to be associated with the downregulation of connecting tissue growth factor (CTGF) and cysteine-rich angiogenic inducer (Cyr) 61 expression, and possibly implicated tumor growth factor (TGF)- $\beta$-mediated signaling pathways.

\section{Materials and methods}

Reagents.MTT and dimethyl sulphoxide(DMSO) were obtained from Sigma-Aldrich (Merck KGaA, Darmstadt, Germany). 
Transwell chambers were purchased from Corning Inc. (Corning, NY, USA). Anti-TAZ (catalog no. 4883) primary antibodies were obtained from Cell Signaling Technology, Inc. (Danvers, MA, USA); anti-CTGF (catalog no. SC-365970), anti-Cyr61 (catalog no. SC-374129), anti-mothers against decapentaplegic homolog (Smad) 3 (catalog no. SC-101154) and anti-Smad4 (catalog no. SC-7966) primary antibodies were purchased from Santa Cruz Biotechnology, Inc. (Dallas, TX, USA); and anti-GAPDH primary antibody was purchased from Sigma-Aldrich (catalog no. G8795; Sigma-Aldrich; Merck $\mathrm{KGaA})$.

Cell isolation and culture. hDPSCs were isolated from healthy third molars or premolars of healthy human subjects (male; age, 16-30 years; $n=12$ ). Individual dental pulps were minced into small pieces $\left(1 \mathrm{~mm}^{3}\right)$ and digested using type I collagenase $(3.0 \mathrm{mg} / \mathrm{ml})$ and dispase $(4.0 \mathrm{mg} / \mathrm{ml}$; Sigma-Aldrich; Merck $\mathrm{KGaA}$ ) for $45 \mathrm{~min}$ at $37^{\circ} \mathrm{C}$. The solution was filtered through a $70-\mathrm{mm}$ cell strainer. Single-cell suspensions were obtained and seeded into $35 \mathrm{~mm}$ culture dishes at a density of 1x10 $/ \mathrm{ml}$. Cells were cultured in Dulbecco's modified Eagle's medium (DMEM; Gibco; Thermo Fisher Scientific, Inc., Waltham, MA, USA), supplemented with $10 \%$ fetal bovine serum (FBS; Hyclone; GE Healthcare Life Sciences, Logan, UT, USA), $2.0 \mathrm{mM} / 1$ glutamine (Invitrogen; Thermo Fisher Scientific, Inc.), and $100 \mathrm{IU} / \mathrm{ml}$ penicillin and streptomycin, and maintained at $37^{\circ} \mathrm{C}$ in a humidified $5 \% \mathrm{CO}_{2}$ atmosphere. When confluent, cells were collected by trypsinization $(0.2 \%$ trypsin and $0.02 \%$ EDTA) and subcultured with DMEM supplemented with $10 \%$ FBS. The medium was replaced every 2-3 days. Cells between 3 and 5 passages were used in experiments.

The present study was approved by the Ethics Committee of the Second Hospital of Hebei Medical University (Shijiazhuang, China). Written informed consent was obtained from all human subjects prior to enrollment in the present study.

Immunofluorescence. A total of $1 \times 10^{5} / \mathrm{ml}$ hDPSCs were seeded onto uncoated coverslips and were fixed with $4 \%$ paraformaldehyde in PBS for $20 \mathrm{~min}$ at room temperature. Fixed samples were permeabilized using $0.3 \%$ Triton X-100 (Sigma-Aldrich; Merck KGaA) in PBS for $15 \mathrm{~min}$ at room temperature. Following blocking with $10 \%$ normal goat serum diluted with $1 \%$ immunoglobulin $\mathrm{G}$ ( $\mathrm{IgG}$ )-free bovine serum albumin (Jackson ImmunoResearch Laboratories, Inc., West Grove, PA, USA) and 0.3\% Triton X-100 in PBS at $37^{\circ} \mathrm{C}$ for $2 \mathrm{~h}$, the samples were incubated with rabbit anti-TAZ polyclonal antibody (1:200) overnight at $4^{\circ} \mathrm{C}$, and with DyLight ${ }^{\mathrm{TM}}$ 488-conjugated goat anti-rabbit IgG (catalog no. 611-141-002; 1:500; Jackson ImmunoResearch Laboratories, Inc.) for $2 \mathrm{~h}$ at room temperature. A total of $5 \mu \mathrm{g} / \mathrm{ml}$ Hoechst 33342 was used to stain the nuclei at $37^{\circ} \mathrm{C}$ for $15 \mathrm{~min}$. Fluorescently-labeled cells were observed under an upright fluorescence microscope (magnification, x20; Olympus Corporation, Tokyo, Japan).

Small interfering (si)RNA transfection. siRNA duplex oligonucleotides targeting TAZ mRNA (siTAZ) and non-targeting duplex oligonucleotides used as negative controls (siCON) were synthesized by Invitrogen (Thermo Fisher Scientific, Inc.). Sequences of siTAZ and siCON were as follows: siTAZ sense, 5'-GGCCAGAGAUAUUUCCUUATT-3'; anti-sense, 5'-UAAGGAAAUAUCUCUGGCCTT-3'; and siCON sense, 5'-UUCACCGAACGUGUCACGUTT-3'; anti-sense: 5'-ACG UGACACGUUCGGAGAATT-3'; Following incubation for $24 \mathrm{~h}$, hDPSCs were transfected with $1 \mu \mathrm{g} / \mathrm{ml}$ of siRNAs, using RNAi-Mate transfection reagent (Shanghai GenePharma Co., Ltd., Shanghai, China), according to the manufacturer's protocol, and cultured in DMEM. Cells were subjected to a second round of transfection prior to subsequent experiments (18).

Reverse transcription-quantitative polymerase chain reaction $(R T-q P C R)$. Following treatment, cells in different groups were harvested and total RNA was extracted using TRIzol ${ }^{\circledR}$ reagent (Invitrogen; Thermo Fisher Scientific, Inc.), according to the manufacturer's protocol. Total RNA was reverse transcribed into cDNA at $42^{\circ} \mathrm{C}$ for $1 \mathrm{~h}$ using Moloney murine leukemia virus reverse transcriptase (GeneCopoeia, Inc., Rockville, MD, USA). qPCR was performed on cDNA using the All-in-One ${ }^{\mathrm{TM}}$ qPCR mix (GeneCopoeia, Inc.) and an ABI7300 Real-Time PCR system (Applied Biosystems; Thermo Fisher Scientific, Inc.). The following primers were obtained from Invitrogen (Thermo Fisher Scientific, Inc.): TAZ: sense, 5'-ATGTTGACC TCCGGACTTTGG-3'; anti-sense: 5'-GAGGAAGGGCTC GCTTTTGT-3'; Cyr61: sense, 5'-GGCAGACCTTGTGAA TATA-3'; anti-sense: 5'-GTATTAGGCTTTATTTACCA-3'; CTGF: sense, 5'-CCCAGACCCAAATATGATT-3'; anti-sense: 5'-CAATGTACGATAGTGCAGT-3'; and GAPDH sense: 5'-AGTCCAACGGCACAGTCAAGG-3'; anti-sense: 5'-AGC ACCAGCATCACCCAT-3';. Amplification conditions were as follows: Initial denaturation at $95^{\circ} \mathrm{C}$ for $1 \mathrm{~min}$; followed by 40 cycles of $95^{\circ} \mathrm{C}$ for $30 \mathrm{sec}, 60^{\circ} \mathrm{C}$ for $20 \mathrm{sec}$ and $72^{\circ} \mathrm{C}$ for $20 \mathrm{sec}$. The relative expression levels of each gene were normalized to GAPDH expression with $2^{-\Delta \Delta \mathrm{Ct}}$ method (19).

Western blot analysis. Total protein was extracted from hDPSCs using RIPA lysis buffer (C1053; Applygen Technologies, Inc., Beijing, China), according to the manufacturer's protocol. Protein concentrations were determined using a bicinchonic acid protein assay kit (Thermo Fisher Scientific, Inc.). A total of $50 \mu \mathrm{g}$ extracted protein samples were separated by $10 \%$ SDS-PAGE and transferred onto polyvinylidene difluoride membranes. Membranes were blocked with 5\% non-fat milk with TBS containing Tween-20 for $2 \mathrm{~h}$ at $37^{\circ} \mathrm{C}$ and then incubated with anti-TAZ (1:1,000), anti-CTGF (1:200), anti-Cyr61 (1:200), anti-Smad3 (1:200), anti-Smad4B (1:200) and anti-GAPDH $(1: 3,000)$ primary antibodies at $4^{\circ} \mathrm{C}$ overnight. Membranes were subsequently incubated with IRDye $800^{\circledR}$-conjugated secondary antibody (catalog no. 608-445-002; 1:20,000; Rockland Immunochemicals, Inc., Limerick, PA, USA) for $1 \mathrm{~h}$ at $37^{\circ} \mathrm{C}$, followed by scanning with the Odyssey ${ }^{\circledR}$ Infrared Imaging System (LI-COR Biosciences, Lincoln, NE, USA). Data were normalized to GAPDH levels and analyzed with Image-Pro Plus software, version 7.0 (Media Cybernetics, Inc., Rockville, MD, USA).

Cellular proliferation assay. Cellular proliferation was evaluated using the MTT assay. hDPSCs were seeded in 96-well 


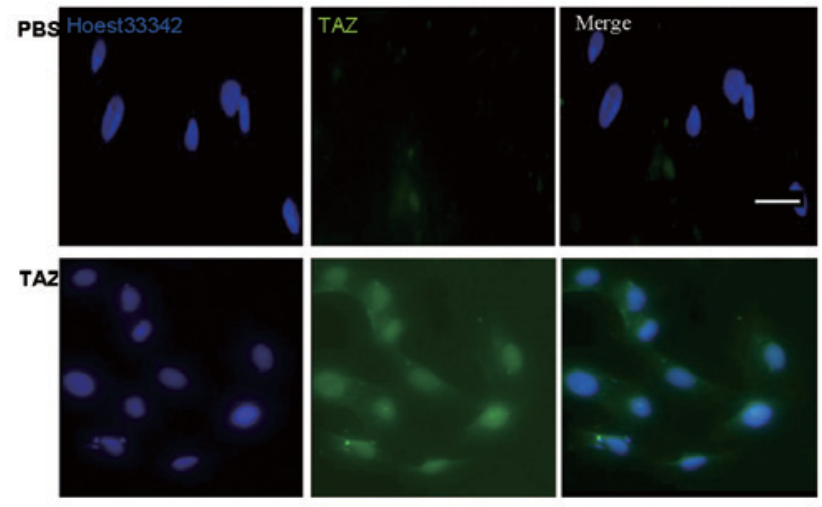

Figure 1. TAZ was expressed in human dental pulp stem cells. TAZ was fluorescently labeled using a rabbit anti-TAZ polyclonal antibody. Cell nuclei were visualized using Hoechst 33342. Scale bar, $50 \mu \mathrm{m}$. Photomicrographs were captured under x20 magnification. TAZ, transcriptional coactivator with PDZ-binding motif.

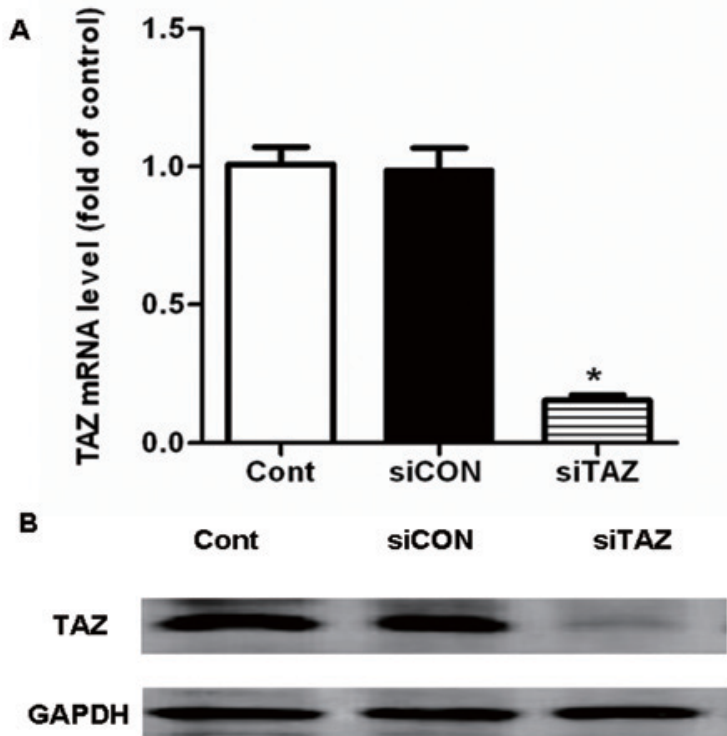

Figure 2. Transfection with siRNA targeting TAZ successfully silences TAZ expression in human dental pulp stem cells. (A) Reverse transcription-quantitative polymerase chain reaction demonstrated the successful transfection. Data are expressed as mean \pm standard deviation. ${ }^{*} \mathrm{P}<0.05$ vs. the siCON group. (B) Representative blot demonstrating reduced TAZ protein expression levels following siRNA transfection. si, small interfering; TAZ, transcriptional coactivator with PDZ-binding motif; Cont, control cells received no treatment; siCON, negative control cells transfected with non-targeting siRNA; siTAZ, cells transfected with siRNA targeting TAZ.

plates, at a density of $3 \times 10^{3}$ cells/well, and incubated at $37^{\circ} \mathrm{C}$ for $24 \mathrm{~h}$. Subsequently, $20 \mu 1 \mathrm{MTT}$ solution $(5 \mathrm{mg} / \mathrm{ml})$ was added and cells were incubated at $37^{\circ} \mathrm{C}$ for $4 \mathrm{~h}$. The culture medium was replaced with DMSO (150 $\mu \mathrm{l} /$ well) and the plates were agitated at room temperature for $10 \mathrm{~min}$ to dissolve the crystals. The optical density at $570 \mathrm{~nm}$ for each well was measured using a microplate reader (Bio-Rad Laboratories, Inc., Hercules, CA, USA).

Cellular proliferation was also assessed via the detection of 5-bromo-2'-deoxyuridine (BrdU)-labeled DNA using the Cell Proliferation ELISA, BrdU kit (Roche Applied Science, Penzberg, Germany). Briefly, hDPSCs were seeded in 96-well plates $\left(3 \times 10^{4}\right.$ cells/well) and were incubated for $24 \mathrm{~h}$ at $37^{\circ} \mathrm{C}$ in the presence of siTAZ or siCON. Subsequently, cells were labeled with $\mathrm{BrdU}$ at $37^{\circ} \mathrm{C}$ for $1 \mathrm{~h}$. BrdU-labeled DNA was quantified using ELISA.

Cellular migration assay. Cellular migration was assessed using a wound healing assay. hDPSCs were seeded in 6-well plates $\left(\sim 5 \times 10^{4}\right.$ cells/well $)$ and cultured to form a monolayer. The cell monolayer was scratched with a $200-\mu 1$ pipette tip. Cells were cultured for $24 \mathrm{~h}$ and wound healing was observed at 0 and $24 \mathrm{~h}$ post-wound infliction under a phase-contrast microscope (Olympus Corporation). Healing was assessed using Scion Image Software, version 4.03 (Scion Corporation, Frederick, Maryland, USA). Cellular invasion was also assessed using Transwell inserts coated with Matrigel (BD Biosciences, Franklin Lakes, NJ, USA). Following treatment, hDPSCs were seeded into the upper chambers at a density of 5x104 cells in $200 \mu \mathrm{l}$ serum-free DMEM; culture medium supplemented with $10 \%$ FBS was added to the lower chambers. Following incubation for $24 \mathrm{~h}$ at $37^{\circ} \mathrm{C}$, the cells on the upper surface of the membrane were removed. The invaded cells on the lower membrane were fixed for 10 min with $4 \%$ paraformaldehyde at $4^{\circ} \mathrm{C}$, stained at $37^{\circ} \mathrm{C}$ for $10 \mathrm{~min}$ with $5 \mu \mathrm{g} / \mathrm{ml}$ Hoechst 33342 and counted under an upright fluorescence microscope (Olympus Corporation).

Treatment with recombinant human thrombospondin 1 $(r$-hTHBS1). hDPSCs were seeded into 6-well plates at a density of $2 \times 10^{5}$ cells/well and cultured for $24 \mathrm{~h}$. Subsequently, cells were incubated at $37^{\circ} \mathrm{C}$ for 10 min with r-hTHBS1, a TGF- $\beta$ activator $(200 \mathrm{ng} / \mathrm{ml}$; R\&D Systems, Inc., Minneapolis, MN, USA). Cells were harvested following treatment and used for further analysis.

Statistical analysis. The statistical significance of the difference between groups was assessed by the Student's t-test for pair-wise comparisons or a one-way analysis of variance, followed by a post hoc Student-Newman-Keuls test for multiple comparisons. Data are expressed as the mean \pm standard deviation. The experiment was repeated three times. $\mathrm{P}<0.05$ was considered to indicate a statistically significant difference. The analysis was performed using SPSS software (version 13.0; SPSS, Inc., Chicago, IL, USA).

\section{Results}

TAZ is expressed in hDPSCs. hDPSCs were cultured for $48 \mathrm{~h}$, fixed and processed for immunofluorescence. TAZ was revealed to be expressed in hDPSCs (Fig. 1).

siTAZ transfection decreases TAZ expression in hDPSCs. Successful transfection with siTAZ in hDPSCs was confirmed using RT-qPCR and western blot analysis. The present results suggested that TAZ mRNA and protein expression levels were significantly decreased following transfection with siTAZ compared with control cells and cells transfected with siCON (Fig. 2).

siTAZ inhibits hDPSC proliferation in vitro. hDPSCs were transfected with siTAZ to silence TAZ expression. Results of the MTT assay demonstrated that TAZ silencing significantly 


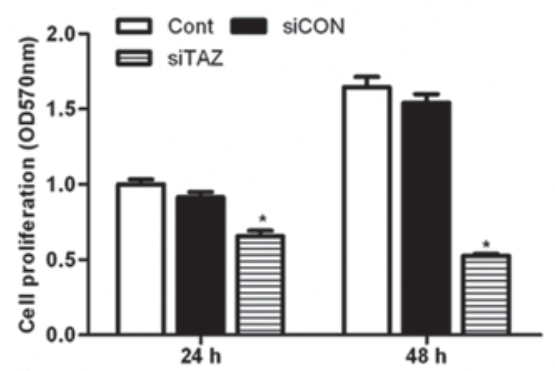

c

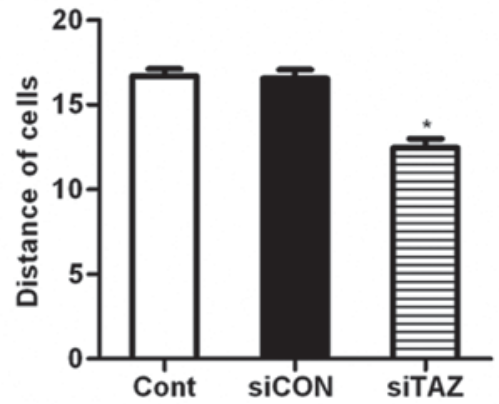

B

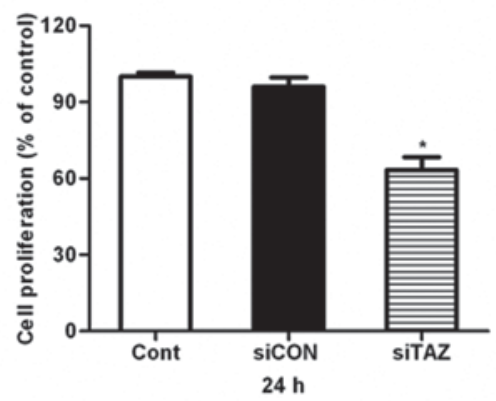

D

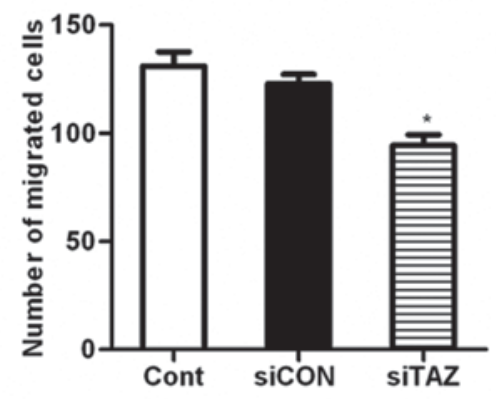

Figure 3. TAZ silencing impaired the proliferative and migratory capabilities of human dental pulp stem cells. Cellular viability following transfection with siRNA targeting TAZ was assessed using (A) MTT and (B) 5-bromo-2'-deoxyuridine assays. Cellular migration following siRNA transfection was evaluated using (C) wound healing and (D) Transwell assays. Data are expressed as the mean \pm standard deviation. " $\mathrm{P}<0.05$ vs. the siCON group. TAZ, transcriptional coactivator with PDZ-binding motif; si, small interfering; Cont, control cells received no treatment; siCON, negative control cells transfected with non-targeting siRNA; siTAZ, cells transfected with siRNA targeting TAZ; OD, optical density.

A
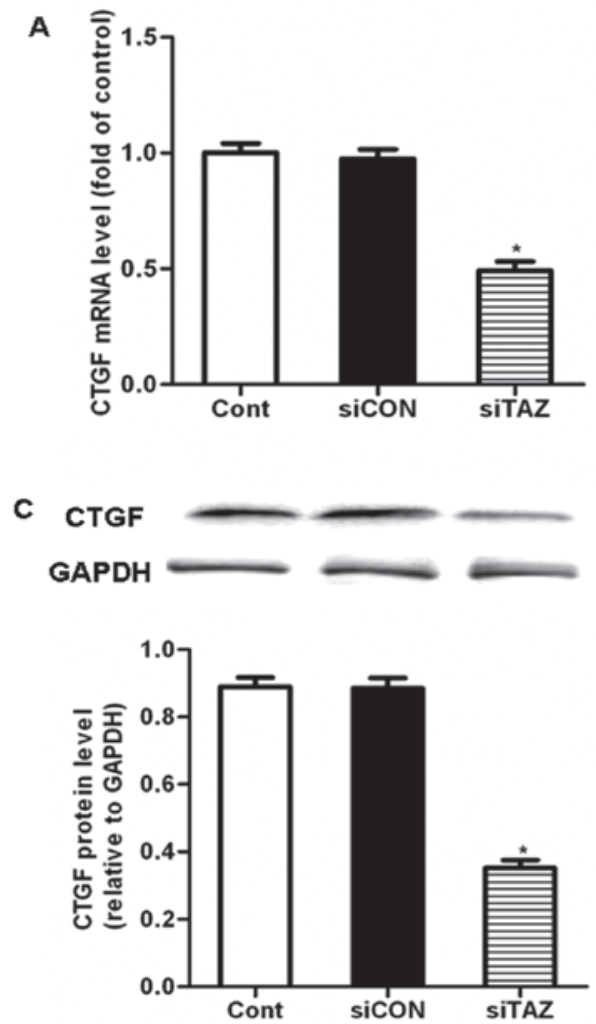

B

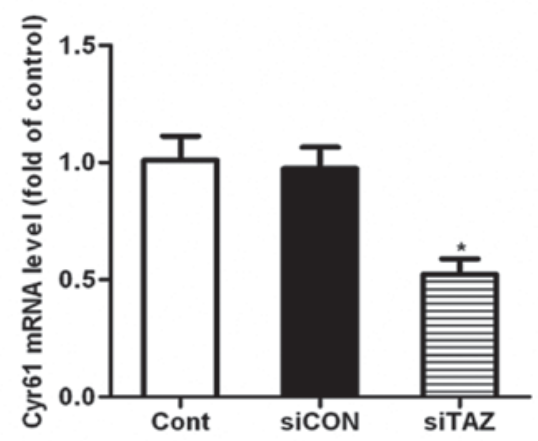

D
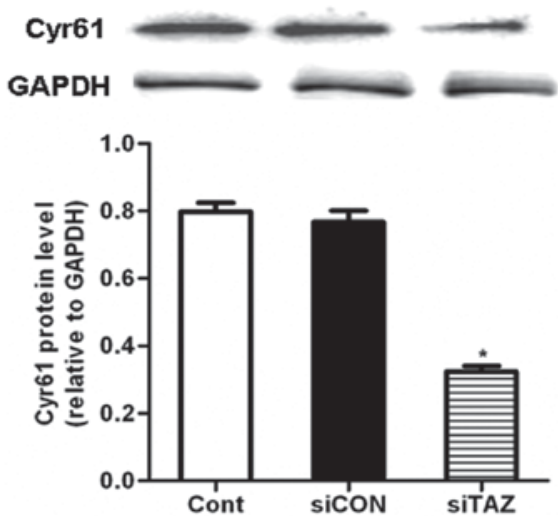

Figure 4. TAZ silencing downregulated CTGF and Cyr61 expression in human dental pulp stem cells. Reverse transcription-quantitative polymerase chain reaction demonstrated that (A) CTGF and (B) Cyr61 mRNA expression levels were significantly downregulated $24 \mathrm{~h}$ post-transfection with siRNA targeting TAZ. Western blot analysis demonstrated that (C) CTGF and (D) Cyr61 protein expression levels were significantly downregulated $24 \mathrm{~h}$ post-siRNA transfection. Data are expressed as the mean \pm standard deviation of 3 independent experiments. ${ }^{*} \mathrm{P}<0.05$ vs. the siCON group. TAZ, transcriptional coactivator with PDZ-binding motif; CTGF, connecting tissue growth factor; Cyr61, cysteine-rich angiogenic inducer 61; si, small interfering; Cont, control cells received no treatment; siCON, negative control cells transfected with non-targeting siRNA; siTAZ, cells transfected with siRNA targeting TAZ. 
A

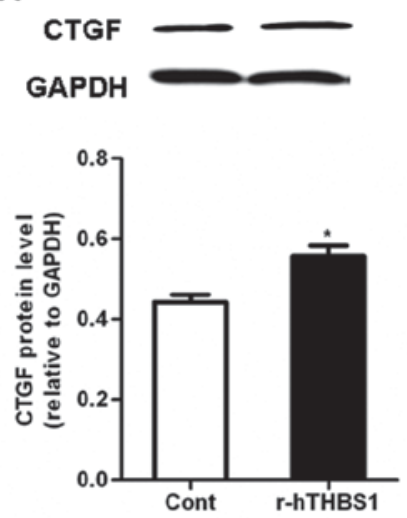

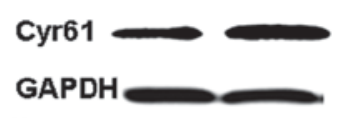

c

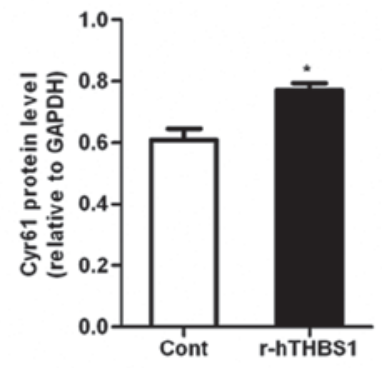

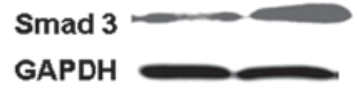

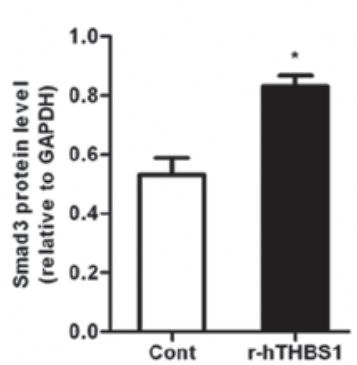

D

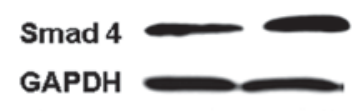

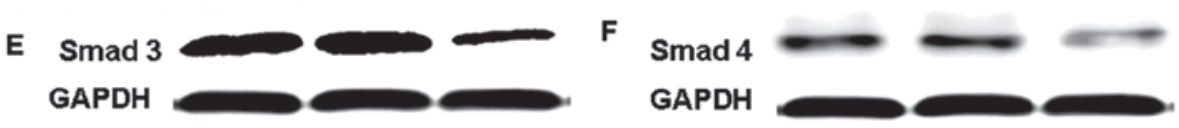
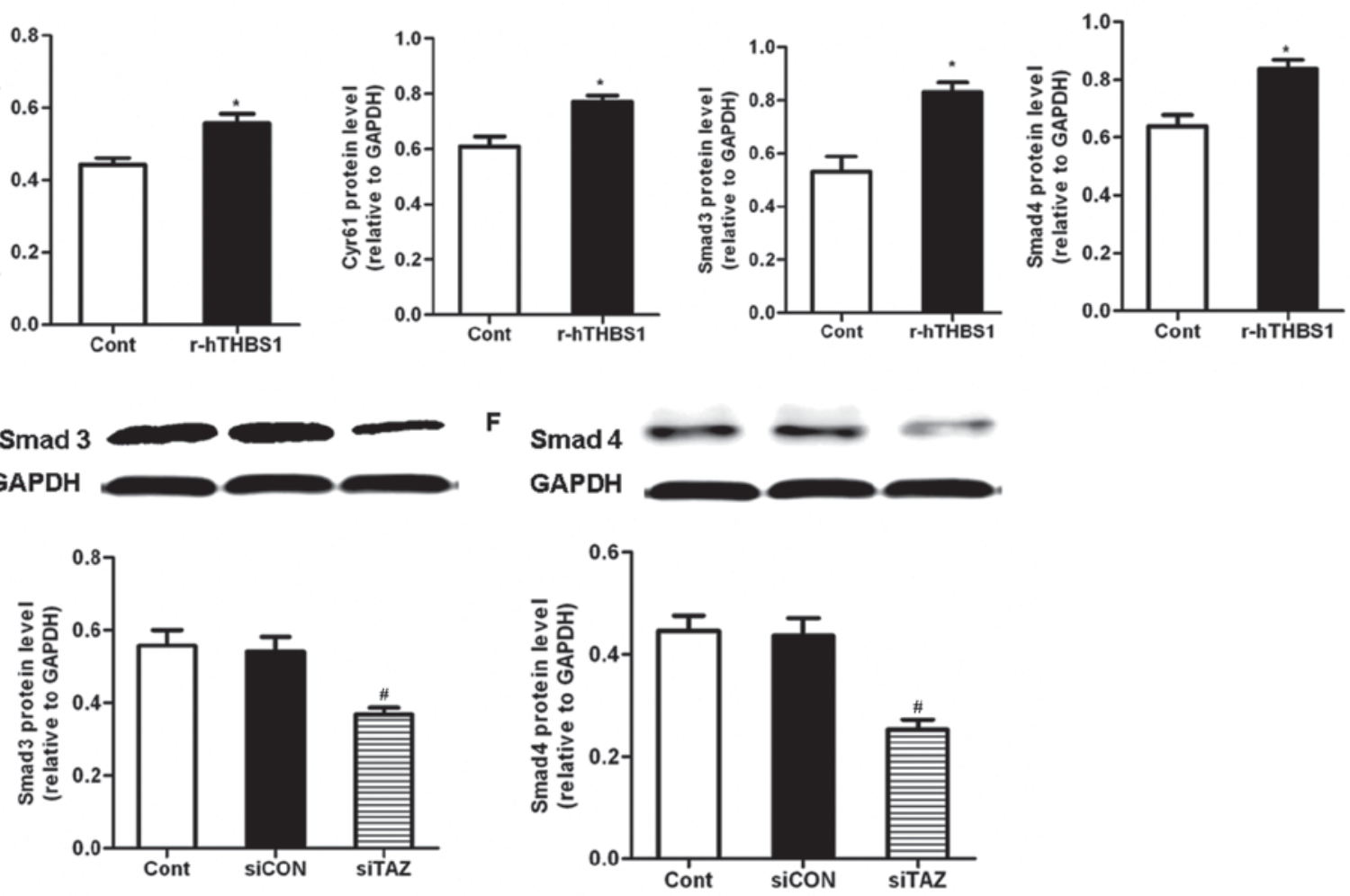

Figure 5. TAZ modulated CTGF and Cyr61 expression via the activation of transforming growth factor- $\beta$-mediated pathways. Western blot analysis was used to assess the protein expression levels of (A) CTGF, (B) Cyr61, (C) Smad3 and (D) Smad4 in hDPSCs following treatment with r-hTHBS1. Data are expressed as the mean \pm standard deviation of 3 independent experiments. ${ }^{*}<0.05$ vs. the Cont group. Protein expression levels of (E) Smad 3 and (F) Smad4 in hDPSCs following transfection with siRNA targeting TAZ. Data are expressed as the mean \pm standard deviation of 3 independent experiments. ${ }^{~} \mathrm{P}<0.05 \mathrm{vs}$. the siCON group. TAZ, transcriptional coactivator with PDZ-binding motif; CTGF, connecting tissue growth factor; Cyr61, cysteine-rich angiogenic inducer 61; hDPSC, human dental pulp stem cell; r-hTHBS, recombinant human thrombospondin; si, small interfering; Cont, control cells received no treatment; siCON, negative control cells transfected with non-targeting siRNA; siTAZ, cells transfected with siRNA targeting TAZ.

inhibited hDPSC proliferation compared with control cells and cells transfected with siCON (Fig. 3A). Similarly, the results of the BrdU assay revealed that transfection with siTAZ decreased hDPSC proliferation in vitro (Fig. 3B).

siTAZ inhibits hDPSC migration in vitro. A wound-healing assay demonstrated that the migration distance of hDPSCs transfected with siTAZ following a scratch wound was significantly reduced compared with control cells and cells transfected with siCON (Fig. 3C). These results suggested that TAZ silencing significantly impaired the migratory capabilities of hDPSCs. Similarly, the results of the Transwell invasion assay revealed that transfection with siTAZ suppressed hDPSC invasion in vitro (Fig. 3D).

siTAZ inhibits hDPSC proliferation and migration through the downregulation of CTGF and Cyr61 expression. To explore the molecular mechanism underlying the inhibitory effects of TAZ silencing on hDPSC proliferation and migration, putative downstream targets of TAZ were investigated. CTGF and Cyr61 have been suggested to be downstream targets of TAZ (20), which may be implicated in the regulation of cellular proliferation, migration and apoptosis. Treatment of hDPSCs with siTAZ for $24 \mathrm{~h}$ significantly decreased the mRNA and protein expression levels of CTGF and Cyr61, compared with control cells and cells transfected with siCON (Fig. 4). These results indicated that siTAZ inhibited hDPSC proliferation and migration through the modulation of CTGF and Cyr61 expression.

siTAZ inhibits CTGF and Cyr61 expression via a TGF- $\beta$-mediated pathway. To further explore the molecular mechanisms underlying the implication of TAZ in the regulation of CTGF and Cyr61 expression, the TGF- $\beta$ pathway was investigated. Following treatment with r-hTHBS1, the protein expression levels of Smad3, Smad4, CTGF and Cyr61 in hDPSCs were significantly upregulated compared with the control (Fig. 5A-D). These results suggested that TAZ may be involved in the regulation of CTGF and Cyr61 expression via a TGF- $\beta$-mediated pathway. Therefore, the expression of Smad3/4 in hDPSCs was investigated following silencing of TAZ expression. Western blot analysis demonstrated that Smad3/4 protein expression levels were significantly downregu lated in TAZ-depleted cells compared with in control cells and cells transfected with siCON (Fig. 5E and F). These results suggested that TAZ may be implicated in TGF- $\beta$-dependent 
pathways that may modulate the expression of CTGF and Cyr61.

\section{Discussion}

The potential applications of human stem cells isolated from dental pulp in tissue regeneration are promising, as hDPSCs may be easily isolated from discarded teeth following extraction. The procedure is non-invasive and lacking ethical concerns, whereas isolated cells may be stored for future use $(21,22)$. Furthermore, harvesting stem cells from patients dental tissue is a reasonable and simple alternative to MSC harvesting (17). Therefore, hDPSCs were used in the present study for in vitro experiments.

TAZ is a transcriptional factor that has been implicated in the development of various types of human tissue $(11,23)$. It serves critical roles in cellular proliferation and apoptosis, as well as in the regulation of organ size $(24,25)$. Previous studies have reported that TAZ overexpression enhanced the proliferation, migration, transformation and epithelial-to-mesenchymal transition of immortalized mammary epithelial cells; conversely, silencing TAZ impaired the proliferative and migratory capabilities of mammary epithelial cells $(26,27)$. Notably, hDPSCs have been demonstrated to express TAZ (17). However, the effects of TAZ on the proliferation and migration of hDPSCs have yet to be elucidated. The present study investigated the roles of TAZ in hDPSCs. Cellular proliferation assays demonstrated that following TAZ knockdown, hDPSC proliferation was impaired. In addition, in vitro transwell and wound healing assays revealed that following TAZ silencing, the migratory and invasive capabilities of hDPSCs were markedly impaired. These results suggested that TAZ may be involved in the regulation of hDPSC proliferation and migration.

The implication of TAZ in processes of cellular proliferation has previously been reported (28); however, the molecular mechanisms underlying the role of TAZ in hDPSCs remain to be elucidated. CTGF and Cyr61 have been identified to be downstream transcriptional targets of TAZ, and have been reported to serve diverse roles in several cellular processes, including development, differentiation, proliferation, adhesion and migration, as well as angiogenesis and tumorigenesis (20,29-31). Furthermore, TAZ, through the activation of its target genes CTGF and Cyr61, has been demonstrated to induce cellular differentiation (32). In the present study, RT-qPCR and western blot analysis revealed that CTGF and Cyr61 mRNA and protein expression levels were downregulated following TAZ silencing in hDPSCs. These results suggested that the mechanisms underlying the actions of TAZ on hDPSC proliferation and migration may involve the modulation of the expression of its downstream target genes CTGF and Cyr61.

It has previously been reported that TGF- $\beta$-mediated pathways are critical in the regulation of tumor formation and invasion (33), whereas CTGF and Cyr61 have been identified to be target genes in TGF- $\beta$ signaling (34). Smad3 and Smad4 have also been reported to participate in TGF- $\beta$-mediated processes (28). The present results suggested that the activity of TGF- $\beta$ pathways was impaired following TAZ depletion.
In conclusion, the results of the present study suggested that TAZ may regulate the proliferation and migration of hDPSCs. In addition, TAZ appeared to exert its regulatory actions on CTGF and Cyr61 expression through TGF- $\beta$-mediated pathways. Therefore, it may be hypothesized that TAZ has potential to be a target for tissue engineering applications.

\section{Acknowledgements}

The present study was supported by the Second Hospital of Hebei Medical University (grant no. 2h1201504).

\section{References}

1. Karaöz E, Demircan PC, Sağlam O, Aksoy A, Kaymaz F and Duruksu G: Human dental pulp stem cells demonstrate better neural and epithelial stem cell properties than bone marrow-derived mesenchymal stem cells. Histochem Cell Biol 136: 455-473, 2011.

2. Gronthos S, Mankani M, Brahim J, Robey PG and Shi S: Postnatal human dental pulp stem cells (DPSCs) in vitro and in vivo. Proc Natl Acad Sci USA 97: 13625-13630, 2000.

3. Liu P, Cai J, Dong D, Chen Y, Liu X, Wang Y and Zhou Y: Effects of SOX2 on proliferation, migration and adhesion of human dental pulp stem cells. PLoS One 10: e0141346, 2015.

4. Dieterich LC, Huang H, Massena S, Golenhofen N, Phillipson $\mathrm{M}$ and Dimberg $\mathrm{A}$ : $\alpha \mathrm{B}$-crystallin/HspB5 regulates endothelial-leukocyte interactions by enhancing $\mathrm{NF}-\kappa \mathrm{B}$-induced up-regulation of adhesion molecules ICAM-1, VCAM-1 and E-selectin. Angiogenesis 16: 975-983, 2013.

5. Grudzinska MK, Kurzejamska E, Bojakowski K, Soin J, Lehmann MH, Reinecke H, Murry CE, Soderberg-Naucler C and Religa P: Monocyte chemoattractant protein 1-mediated migration of mesenchymal stem cells is a source of intimal hyperplasia. Arterioscler Thromb Vasc Biol 33: 1271-9, 2013.

6. Luo Z, Li D, Kohli MR, Yu Q, Kim S and He WX: Effect of Biodentine $^{\mathrm{TM}}$ on the proliferation, migration and adhesion of human dental pulp stem cells. J Dent 42: 490-497, 2014.

7. Tate MC, Garcia AJ, Keselowsky BG, Schumm MA, Archer DR and LaPlaca MC: Specific beta1 integrins mediate adhesion, migration and differentiation of neural progenitors derived from the embryonic striatum. Mol Cell Neurosci 27: 22-31, 2004.

8. Yang D, Sun S, Wang Z, Zhu P, Yang Z and Zhang B: Stromal cell-derived factor-1 receptor $\mathrm{CXCR} 4$-overexpressing bone marrow mesenchymal stem cells accelerate wound healing by migrating into skin injury areas. Cell Reprogram 15: 206-215, 2003.

9. Kanai F, Marignani PA, Sarbassova D, Yagi R, Hall RA, Donowitz M, Hisaminato A, Fujiwara T, Ito Y, Cantley LC and Yaffe MB: TAZ: A novel transcriptional co-activator regulated by interactions with 14-3-3 and PDZ domain proteins. EMBO J 19: 6778-6791, 2000.

10. Murakami M, Tominaga J, Makita R, Uchijima Y, Kurihara Y, Nakagawa O, Asano $\mathrm{T}$ and Kurihara $\mathrm{H}$ : Transcriptional activity of Pax3 is co-activated by TAZ. Biochem Biophys Res Commun 339: 533-539, 2006.

11. Hong JH, Hwang ES, McManus MT, Amsterdam A, Tian Y, Kalmukova R, Mueller E, Benjamin T, Spiegelman BM, Sharp PA, et al: TAZ, a transcriptional modulator of mesenchymal stem cell differentiation. Science 309: 1074-1078, 2005.

12. Murakami M, Nakagawa M, Olson EN and Nakagawa O: A WW domain protein TAZ is a critical coactivator for TBX5, a transcription factor implicated in Holt-Oram syndrome. Proc Natl Acad Sci USA 102: 18034-18039, 2005.

13. Park KS, Whitsett JA, Di Palma T, Hong JH, Yaffe MB and Zannini M: TAZ interacts with TTF-1 and regulates expression of surfactant protein-C. J Biol Chem 279: 17384-17390, 2004.

14. Pan D: The hippo signaling pathway in development and cancer. Dev Cell 19: 491-505, 2010.

15. Hong JH and Yaffe MB: TAZ: A beta-catenin-like molecule that regulates mesenchymal stem cell differentiation. Cell Cycle 5: 176-179, 2006.

16. Jeong H, Bae S, An SY, Byun MR, Hwang JH, Yaffe MB, Hong JH and Hwang ES: TAZ as a novel enhancer of MyoD-mediated myogenic differentiation. FASEB J 24: 3310-3320, 2010. 
17. Suh JS, Kim KS, Lee JY, Choi YJ, Chung CP and Park YJ: A cell-permeable fusion protein for the mineralization of human dental pulp stem cells. J Dent Res 91: 90-96, 2012.

18. Xue P, Wu X, Zhou L, Ma H, Wang Y, Liu Y, Ma J and Li Y: IGF1 promotes osteogenic differentiation of mesenchymal stem cells derived from rat bone marrow by increasing TAZ expression. Biochem Biophys Res Commun 433: 226-231, 2013.

19. Livak KJ and Schmittgen TD: Analysis of relative gene expression data using real-time quantitative PCR and the 2(-Delta Delta C(T)) Method. Methods 25: 402-408, 2001.

20. Lai D, Ho KC, Hao Y and Yang X: Taxol resistance in breast cancer cells is mediated by the hippo pathway component TAZ and its downstream transcriptional targets Cyr61 and CTGF. Cancer Res 71: 2728-2738, 2011.

21. Iohara K, Zheng L, Wake H, Ito M, Nabekura J, Wakita H, Nakamura $\mathrm{H}$, Into T, Matsushita $\mathrm{K}$ and Nakashima M: A novel stem cell source for vasculogenesis in ischemia: Subfraction of side population cells from dental pulp. Stem Cells 26 : N2408-N2418, 2008.

22. Nakamura S, Yamada Y, Katagiri W, Sugito T, Ito K and Ueda M: Stem cell proliferation pathways comparison between human exfoliated deciduous teeth and dental pulp stem cells by gene expression profile from promising dental pulp. J Endod 35: $1536-1542,2009$.

23. Hong W and Guan KL: The YAP and TAZ transcription co-activators: Key downstream effectors of the mammalian Hippo pathway. Semin Cell Dev Biol 23: 785-793, 2012.

24. Wang K, Degerny C, Xu M and Yang XJ: YAP, TAZ and Yorkie: A conserved family of signal-responsive transcriptional coregulators in animal development and human disease. Biochem Cell Biol 87: 77-91, 2009.

25. Zeng Q and Hong W: The emerging role of the hippo pathway in cell contact inhibition, organ size control, and cancer development in mammals. Cancer Cell 13: 188-192, 2008.

26. Chan SW, Lim CJ, Guo K, Ng CP, Lee I, Hunziker W, Zeng Q and Hong W: A role for TAZ in migration, invasion, and tumorigenesis of breast cancer cells. Cancer Res 68: 2592-2598, 2008.
27. Lei QY, Zhang H, Zhao B, Zha ZY, Bai F, Pei XH, Zhao S, Xiong Y and Guan KL: TAZ promotes cell proliferation and epithelial-mesenchymal transition and is inhibited by the hippo pathway. Mol Cell Biol 28: 2426-2436, 2008.

28. Wang Q, Xu Z, An Q, Jiang D, Wang L, Liang B and Li Z TAZ promotes epithelial to mesenchymal transition via the upregulation of connective tissue growth factor expression in neuroblastoma cells. Mol Med Rep 11: 982-988, 2015.

29. Dhar A and Ray A: The CCN family proteins in carcinogenesis. Exp Oncol 32: 2-9, 2010.

30. Leask A and Abraham DJ: All in the CCN family: Essential matricellular signaling modulators emerge from the bunker. J Cell Sci 119: 4803-4810, 2006.

31. Leivonen SK and Kähäri VM: Transforming growth factor-beta signaling in cancer invasion and metastasis. Int J Cancer 121: 2119-2124, 2007.

32. Kim KM, Choi YJ, Hwang JH, Kim AR, Cho HJ, Hwang ES, Park JY, Lee SH and Hong JH: Shear stress induced by an interstitial level of slow flow increases the osteogenic differentiation of mesenchymal stem cells through TAZ activation. PLoS One 9: e92427, 2014

33. Labbé E, Lock L, Letamendia A, Gorska AE, Gryfe R, Gallinger S, Moses HL and Attisano L: Transcriptional cooperation between the transforming growth factor-beta and Wnt pathways in mammary and intestinal tumorigenesis. Cancer Res 67: 75-84, 2007.

34. Xie JJ, Xu LY, Wu JY, Shen ZY, Zhao Q, Du ZP, Lv Z, Gu W, Pan F, Xu XE, et al: Involvement of CYR61 and CTGF in the fascin-mediated proliferation and invasiveness of esophageal squamouscell cell carcinomas cells. Am J Pathol 76: 939-951, 2010 . 